\title{
EFICACIA IN VITRO DE EXTRACTOS DEL HONGO COMESTIBLE PLEUROTUS OSTREATUS KUMM PARA EL CONTROL DE SITOPHILUS ZEAMAIS MOTSCHULSKY
}

\section{IN VITRO EFFICACY OF EDIBLE MUSHROOM PLEUROTUS OSTREATUS KUMM EXTRACTS FOR SITOPHILUS ZEAMAIS MOTSCHULSKY CONTROL}

\author{
Valentina Pino ${ }^{1}$, Gonzalo Silva-Aguayo ${ }^{1 *}$, Inés Figueroa-Cares ${ }^{1}$, Macarena Gerding-González ${ }^{1}$, \\ Patricia Loyola ${ }^{1}$, Gloria S. Castañeda-Ramirez ${ }^{2}$, Liliana Aguilar-Marcelino ${ }^{2 *}$ \\ ${ }^{1}$ Departamento de Producción Vegetal, Facultad de Agronomía, Universidad de Concepción, Vicente \\ Méndez 595, Chillán, Chile. \\ ${ }^{2}$ Centro Nacional de Investigación Disciplinaria en Salud Animal e Inocuidad, INIFAP, Km 11 Carretera \\ Federal Cuernavaca-Cuautla, Jiutepec, Estado de Morelos, México. \\ *Autores para correspondencia E-mail: gosilva@udec.cl; aguilar.liliana@inifap.gob.mx
}

\section{RESUMEN}

El gorgojo del maíz, Sitophilus zeamais Motschulsky, es a nivel mundial una de las plagas más importantes de los cereales almacenados. Actualmente su manejo se realiza principalmente con insecticidas sintéticos, con escasa variación de mecanismos de acción, por lo que una alternativa son los compuestos naturales, especialmente los extractos vegetales. El objetivo de esta investigación fue evaluar, en condiciones de laboratorio, el efecto insecticida/insectistático de extractos de Pleurotus ostreatus Kumm con solventes de distinta polaridad para el control de adultos de S. zeamais. Las variables evaluadas fueron toxicidad por contacto y fumigación, repelencia y antixenosis, con un diseño experimental completamente al azar. La mayor toxicidad se obtuvo por fumigación con el extracto en acetato de etilo con una mortalidad de $100 \%$ en la concentración $30 \mu \mathrm{L}$ en $0,15 \mathrm{~L}^{-1}$ aire y una $\mathrm{CL}_{50}$ de $18,3 \mu \mathrm{L}$ en $0,15 \mathrm{~L}^{-1}$ aire. Los extractos en acetato de etilo y agua destilada mostraron actividad repelente y todos registraron efecto de antixenosis. Se concluyó que el extracto de $P$. ostreatus en acetato de etilo presenta actividad fumigante, repelente y antixenótica sobre adultos de S. zeamais.

Palabras clave: Gorgojo del maíz, Sitophilus zeamais, hongos comestibles, Pleurotus ostreatus, pesticida, control de pestes

\section{ABSTRACT}

The maize weevil, Sitophilus zeamais Motschulsky, is one of the most important pests of stored cereals worldwide. Currently, it is mainly controlled with the use of synthetic insecticides, with poor variation of action mechanisms. Natural compounds, particularly plant extracts, are an alternative to control this pest. The objective of this study was to evaluate, under laboratory conditions, the insecticidal/insectistatic effect of extracts with different polarity solvents of Pleurotus ostreatus Kumm against $S$. zeamais adult. The variables evaluated were contact and fumigant toxicity, repellency and antixenosis. A completely randomized experimental design was used. The highest toxicity was obtained by fumigation with ethyl acetate extract with a mortality of $100 \%$ at a concentration of 30 $\mu \mathrm{L} 0.15 \mathrm{~L}^{-1}$ air, and a $\mathrm{CL}_{50}$ of $18,3 \mu \mathrm{L}$ at $0,15 \mathrm{~L}^{-1}$ air. Extracts in ethyl acetate and distilled water showed

\footnotetext{
Recibido: 16 octubre 2019. Aceptado: 03 noviembre 2019.
} 
repellent activity, while all extracts exhibited antixenosis effects. It can be concluded that $P$. ostreatus extract in ethyl acetate has fumigant, repellent and antixenotic activity against $S$. zeamais adult.

Key words: Maize weevil, Sitophilus zeamais, edible mushrooms, Pleurotus ostreatus, pesticides, pest control

\section{INTRODUCCIÓN}

Los cereales son un producto agrícola con significancia económica y social, debido a que se utilizan en la alimentación humana (Almeida et al., 2014) como fuente de carbohidratos y proteínas (García-Lara et al., 2007), e industrialmente para la producción de almidón, aceite, harina, glucosa, productos químicos, raciones para animales y elaboración de formulaciones alimenticias (Almeida et al., 2014). Sin embargo, existen factores que limitan su producción, como son los insectos plaga (Usseglio et al., 2017). El género Sitophilus (Coleoptera: Curculionidae), incluye las plagas más destructivas de los cereales almacenados. El gorgojo del arroz, (Sitophilus oryzae L.), el gorgojo del trigo (Sitophilus granarius L.) y el gorgojo del maíz (Sitophilus zeamais Motsch.) son particularmente abundantes en zonas tropicales, subtropicales y cálidas (Devi et al., 2017). El daño de estas especies lo realizan los adultos y larvas que se alimentan y desarrollan dentro del endosperma de la semilla, dañando el grano completo (Wale and Assegie, 2015). El control se realiza fundamentalmente con aplicaciones continuas de insecticidas de contacto como organofosforados y piretroides (Ortiz et al., 2012), o fumigantes sintéticos como fosfuro de aluminio o bromuro de metilo (Hou et al., 2016). Estos compuestos han tenido un rol importante en el control de plagas de los granos almacenados, por su amplio espectro de control y facilidad de penetración en los cereales, dejando mínimos residuos (Zettler y Arthur, 2000). Sin embargo, su uso irracional ha producido efectos adversos, como contaminación del ambiente, presencia de residuos peligrosos en alimentos, junto con antecedentes de insectos resistentes a insecticidas sintéticos como organofosforados o piretroides (Silva et al., 2003). Por tanto, se hace necesaria la búsqueda de nuevas estrategias que no presenten estos problemas (Pohleven et al., 2011). Una de las opciones son los compuestos de origen natural que han sido utilizados para el control de insectos y ácaros desde muy antigua data, como polvos, extractos o aceites esenciales, siendo estos últimos los más utilizados para el control de plagas de los cereales almacenados (Regnault-Roger, 1997; Kim et al., 2013; Hernández-Lambraño et al., 2015).

Recientemente se ha encontrado que varios hongos comestibles delaclaseBasidiomycetes, han mostrado actividad insecticida (Pohleven et al., 2011). Específicamente la especie de basidiomicete conocida, por sus propiedades medicinales, como hongo ostra (Pleurotus ostreatus Kumm) del cual se han reportado propiedades insecticidas con extractos en solventes de distinta polaridad, como éter de petróleo, metanol-cloroformo y agua, para el control de Tribolium castaneum (Herbst) (Rahman et al., 2011) y Macrosiphum rosae L.; (Hemiptera: Aphididae), registrando mayor toxicidad a medida que aumenta el tiempo de exposición del insecto al extracto. Otra de las propiedades de $P$. ostreatus es el efecto biodegradador de insecticidas organofosforados en el suelo, debido a que secreta varias enzimas extracelulares que degradan una gran variedad de compuestos naturales y antropogénicos, que presentan estructuras químicas similares a la lignina (Ganash et al., 2016).

Finalmente, los hongos comestibles también constituyen una alternativa terapéutica para infecciones causadas por bacterias patógenas vegetales, debido a que las propiedades nutricionales de los basidiomicetes han demostrado ser relevantes en la producción de metabolitos secundarios, con funciones de defensa contra plagas y enfermedades (Carrillo et al., 2017).

El objetivo de la presente investigación fue evaluar, en condiciones de laboratorio, el efecto insecticida como insecticida de contacto o fumigante e insectistático como repelente y antixenótico de extractos de $P$. ostreatus con solventes de distinta polaridad para el control de adultos de $S$. zeamais.

\section{MATERIALES Y MÉTODOS}

El estudio se realizó en el Laboratorio de Entomología de la Facultad de Agronomía de la Universidad de Concepción, Campus Chillán, Región de Nuble, Chile. Se realizaron bioensayos con tratamientos consistentes en extractos de $P$. ostreatus para el control de adultos de S. zeamais. Específicamente los tratamientos consistieron en extractos con solventes de diferente polaridad (hexano $>$ metanol $>$ acetato de etilo $>$ agua destilada) en concentraciones de 0,$5 ; 1,0 ; 2,0 ; y$ $4,0 \%(\mathrm{v} / \mathrm{v})$. 


\section{Insectos}

Los insectos se obtuvieron de colonias permanentes del Laboratorio de Entomología de la Facultad de Agronomía. Las colonias se mantienen en frascos de vidrio de 1 L con maíz como sustrato alimenticio y en condiciones de $30 \pm$ $2^{\circ} \mathrm{C}$ de temperatura, $60 \pm 5 \%$ de humedad relativa y oscuridad total en una cámara bioclimática. Estas colonias se renuevan anualmente con insectos provenientes de cereales infestados y desechados por vendedores del mercado de hortalizas, frutas y granos de la ciudad de Chillán. Las colonias se limpiaron cada siete días extrayendo todos los insectos adultos, los que se utilizaron para iniciar nuevas colonias y la progenie emergida $\left(F_{1}\right)$ se utilizó para los bioensayos.

\section{Cereal}

El maíz para las colonias y bioensayos fue el cultivar Dekalb DK440, que se obtuvo de la Estación Experimental "El Nogal", de la Facultad de Agronomía de la Universidad de Concepción, Campus Chillán, Chile. El maíz no recibió aplicaciones durante la temporada para el control del gusano de la mazorca (Helicoverpa zea Boddie) ni de postcosecha pero con el objetivo de evitar alguna alteración de los resultados de los bioensayos, por cualquier tipo de infestación externa de insectos o residuo de insecticida por deriva de cultivos aledaños, el maíz se lavó con agua potable, se secó a temperatura ambiente (20 $\pm 5^{\circ} \mathrm{C}$ ) por $24 \mathrm{~h}$ y posteriormente se congeló por $48 \mathrm{~h}$.

\section{Extractos}

El hongo ostra (Setas del Huerto, Agrícola Funghi S.A., Fundo Abrantes Hijuela $\mathrm{N}^{\circ} 1$, Paine, Región Metropolitana, Chile) se obtuvo de supermercados de la ciudad de Chillán, Región de Nuble, Chile. Los cuerpos fructíferos de $P$. ostreatus se deshidrataron en una estufa de secado (Memmert Gmbh, UNB 500, Schwabach, Alemania) a $40 \pm 5^{\circ} \mathrm{C}$ de temperatura durante 48 h. Posteriormente la obtención de los extractos se realizó en un sistema Soxhlet, que opera recirculando los vapores condensados desde el solvente, que se encuentra en evaporación continua, arrastrando los principios activos (Caldas, 2012). La extracción se realizó con una batería de solventes de diferente polaridad que fueron: hexano > metanol > acetato de etilo > agua destilada. Posteriormente los extractos se concentraron en un rotavator giratorio y lo obtenido se utilizó como solución madre (100\%) y el solvente puro constituyó testigo en cada bioensayo.

\section{Bioensayos}

\section{Toxicidad por contacto con grano tratado}

Este bioensayo se llevó a cabo con la metodología de Obeng-Oferi y Reichmuth (1997). Se utilizaron envases de vidrio de $500 \mathrm{~mL}$, en el que se mezclaron $100 \mathrm{~g}$ de maíz con una solución de extracto de $P$. ostreatus diluido en $1 \mathrm{~mL}$ del respectivo solvente en concentraciones de 0,5 ; 1,$0 ; 2,0 ;$ y $4,0 \%(\mathrm{v} / \mathrm{v})$, más un testigo consistente en el solvente correspondiente a cada extracto. Los envases se agitaron manualmente por 30 segundos para permitir un cubrimiento uniforme de los granos por la solución, para luego dejarlos a temperatura ambiente por media hora para que se evaporara totalmente el solvente. En seguida, cada envase se infestó con 10 parejas de insectos, los cuales se diferenciaron por sexo de acuerdo al criterio de Halstead (1963), quien señala que el rostrum del macho es de menor longitud, mayor grosor y rugosidad que el de la hembra. Posteriormente, los envases se cubrieron con sus tapas perforadas para permitir el intercambio gaseoso, y almacenaron en una cámara bioclimática (IPS 749, Memmert Gmbh, Schwabach, Germany) a $30 \pm 2^{\circ} \mathrm{C}$ de temperatura, $60 \pm 5 \%$ de humedad relativa y oscuridad total.

La toxicidad de cada tratamiento se evaluó registrando la mortalidad y emergencia de insectos adultos $\left(\mathrm{F}_{1}\right)$, junto con la pérdida de peso y germinación del grano. La mortalidad se evaluó a los 15 días después de la infestación (DDI), cuantificando los insectos vivos y muertos para obtener el porcentaje de mortalidad que se corrigió con la fórmula de Abbott (Abbott, 1925). El porcentaje de emergencia de insectos adultos $\left(\mathrm{F}_{1}\right)$ se evaluó a los $55 \mathrm{DDI}$, contabilizando todos los insectos adultos emergidos después de ese periodo. La pérdida de peso del grano también se evaluó a los 55 DDI, por la diferencia entre el peso inicial (100 g) y final del grano. La prueba de germinación consistió en que durante siete días se colocaron a germinar 20 semillas ( $\sin$ daño aparente) de cada repetición por tratamiento, en placas Petri acondicionadas con un papel filtro húmedo en condiciones de $20 \pm 5^{\circ} \mathrm{C}$ de temperatura en una cámara bioclimática (FOC 225E, Velp Scientifica, Usmate, Italia) (Pérez et al., 2007).

\section{Repelencia}

La metodología para evaluar el efecto repelente se adaptó de Mazzonetto y Vendramim (2003). Se utilizó una arena de libre elección (Choice arena) formada por cinco placas Petri plásticas de $5 \mathrm{~cm}$ de diámetro y $1,5 \mathrm{~cm}$ de altura, estando una placa central conectada a las otras cuatro por tubos plásticos de $10 \mathrm{~cm}$ de longitud dispuestos diagonalmente formando una " $\mathrm{X}$ ". 
En envases de vidrio con 50 g de maíz, se aplicó $1 \mathrm{~mL}$ de solución de extracto de $P$. ostreatus en el respectivo solvente (hexano $>$ metanol $>$ acetato de etilo $>$ agua destilada) en concentraciones de 0,$5 ; 1,0 ; 2,0$ o 4,0\% (v/v) más un testigo constituido por sólo el solvente correspondiente a cada extracto. Luego, cada tratamiento se colocó en dos placas Petri simétricamente opuestas mientras que en las otras dos placas se ubicó el testigo que consistió en el grano mezclado con el solvente. A continuación, en la placa central se liberaron 20 insectos adultos sin diferenciación de sexo, y luego de 24 h se contabilizó el número de insectos por placa. La comparación de los tratamientos se realizó con el índice de repelencia (IR) de Mazzonetto y Vendramim (2003) que clasifica el tratamiento como repelente si IR $<1$, atrayente si IR $>1$ y neutro si IR $=1$. Sin embargo, considerando que $\mathrm{IR}=1$ equivale a un $51 \%$ de insectos repelidos se utilizó la escala complementaria de Bustos et al. (2017) para cuantificar de manera más precisa los tratamientos clasificados como repelentes, la cual indica que los valores de índice de repelencia son: $\geq 1,0$ (Sin repelencia); 0,76-0,99 (Repelencia débil); 0,51-0,75 (Repelencia moderada); 0,26-0,50 (Repelencia alta) y 0,0-0,25 (Repelencia muy alta).

\section{Efecto fumigante}

La toxicidad por fumigación se evaluó con la metodología de Chu et al. (2011), que consiste en impregnar un papel filtro circular de $2,5 \mathrm{~cm}$ de diámetro, con una micropipeta, con el extracto de $P$. ostreatus sin diluir en volúmenes de $5,10,15,20$, 25 y $30 \mu \mathrm{L}$ en $0,15 \mathrm{~L}^{-1}$ (equivalentes a 33,$3 ; 66,6$; $100 ; 133,3 ; 166,6$ y $200 \mu \mathrm{L} \mathrm{L}^{-1}$ aire) más un testigo consistente en el papel filtro sólo con el solvente correspondiente a cada extracto. El papel filtro circular estaba adherido en la cara interior de la tapa de un envase de $150 \mathrm{~mL}$ que contenía 20 g de maíz infestado con 10 insectos adultos sin diferenciación de sexo. Cada tratamiento tuvo cuatro repeticiones y los envases se almacenaron a $30 \pm 2^{\circ} \mathrm{C}$ de temperatura, $60 \pm 5 \%$ de humedad relativa y oscuridad total en una cámara bioclimática. La mortalidad se evaluó a los $5 \mathrm{DDI}$, y los resultados se corrigieron con la fórmula de Abbott (1925).

\section{Antixenosis (No preferencia)}

La antixenosis se evaluó con la metodología descrita por Cruzat et al. (2009) que consiste en una arena de selección, formada por ocho placas Petri plásticas de $5 \mathrm{~cm}$ de diámetro distribuidas alrededor de una placa central y conectadas a esta a través de tubos de $10 \mathrm{~cm}$ de largo y 0,25 $\mathrm{cm}$ de diámetro. En las ocho placas periféricas se depositaron $20 \mathrm{~g}$ de maíz, mezclado con los diferentes tratamientos que fueron: 0,$125 ; 0,25 ; 0,5$;
1,$0 ; 2,0 ; 4,0$ u $8,0 \%$ (v/v) de extracto de P. ostreatus disuelto en $1 \mathrm{~mL}$ del respectivo solvente (hexano $>$ metanol $>$ acetato de etilo $>$ agua destilada). Luego, en la placa central se liberaron 50 insectos adultos sin diferenciación de sexo y a los 5 DDI se contabilizó el número de insectos por placa. Cada arena de selección constituyó una repetición y se montaron 10 de ellas. Con los datos obtenidos se calculó el índice de repelencia de Mazzonetto y Vendramim (2003) complementado con la escala de Bustos et al. (2017).

\section{Diseño experimental}

El diseño experimental fue completamente al azar con un arreglo factorial de $3 \times 4$ (tres solventes y cuatro concentraciones), para el bioensayo de toxicidad por contacto; y de $4 \times 4$ (cuatro solventes y cuatro concentraciones) para el de efecto fumigante. Se realizó un test de normalidad Shapiro-Wilks y de homocedasticidad de Barlett, y los datos que no se ajustaron a una distribución normal se transformaron a arcoseno $(x / 100)^{1 / 2}$ para el análisis estadístico. Los análisis se realizaron con el software estadístico InfoStat, mediante un análisis de varianza (ANOVA) y un test de comparación de medias Tukey $(\mathrm{P} \leq 0,05)$.

\section{RESULTADOS Y DISCUSIÓN}

\section{Toxicidad por contacto con grano tratado}

La toxicidad del extracto con metanol registró una mortalidad máxima de $21,5 \%$, mientras que los extractos con acetato de etilo y hexano no alcanzaron el $17 \%$ de insectos muertos (Tabla 1). El extracto en agua destilada no presentó toxicidad contra los insectos registrando $0 \%$ de insectos muertos. De acuerdo al criterio propuesto por Lagunes (1994), que clasifica como prometedores aquellos tratamientos con una mortalidad superior al $50 \%$, debe señalarse que $P$. ostreatus no presenta toxicidad por contacto significativa contra adultos de $S$. zeamais. Estos resultados no coinciden con Rahman et al. (2011), quienes con extractos de $P$. ostreatus en éter de petróleo, cloroformo y metanol obtuvieron una alta toxicidad contra adultos de $T$. castaneum, la cual se incrementó a medida que aumentó el tiempo de exposición. A pesar de que este autor no indica valores específicos de mortalidad, concluye que $P$. ostreatus es efectivo contra esta plaga en concentraciones entre 5 y $40 \mathrm{mg}$ $\mathrm{mL}^{-1}$, además de clasificarse como un plaguicida natural prometedor. La baja mortalidad obtenida en el presente bioensayo podría deberse al proceso con que se extrajeron los componentes responsables de la actividad insecticida, o a que las concentraciones empleadas y el tiempo de exposición fueron insuficientes.

La emergencia de insectos adultos $\left(F_{1}\right)$ fue 
Tabla 1. Mortalidad y emergencia de adultos de S. zeamais tratados con extractos de P. ostreatus en metanol, hexano y acetato de etilo.

Table 1. Mortality and emergence of adults of $S$. zeamais treated with $P$. ostreatus extracts in methanol, hexane and ethyl acetate.

\begin{tabular}{cccc}
\hline Tratamiento & $\begin{array}{c}\text { Concentración } \\
(\mathbf{\%})(\mathbf{v} / \mathbf{v})\end{array}$ & $\begin{array}{c}\text { Mortalidad } \\
\mathbf{( \% )}\end{array}$ & $\begin{array}{c}\text { Emergencia* } \\
\left(\mathbf{F}_{\mathbf{1}}\right)\end{array}$ \\
\hline Metanol & 0,0 & - & $149 \mathrm{a}$ \\
& 0,5 & $21,5 \mathrm{a}$ & $143 \mathrm{a}$ \\
& 1,0 & $14,0 \mathrm{a}$ & $149 \mathrm{a}$ \\
& 2,0 & $10,3 \mathrm{a}$ & $170 \mathrm{a}$ \\
& 4,0 & $20,4 \mathrm{a}$ & $156 \mathrm{a}$ \\
\hline Hexano & 0,0 & - & $107 \mathrm{a}$ \\
& 0,5 & $16,3 \mathrm{a}$ & $149 \mathrm{a}$ \\
Ac. etilo & 1,0 & $10,0 \mathrm{a}$ & $125 \mathrm{a}$ \\
& 2,0 & $5,0 \mathrm{a}$ & $111 \mathrm{a}$ \\
& 4,0 & $7,5 \mathrm{a}$ & $104 \mathrm{a}$ \\
& 0,0 & - & $54 \mathrm{ab}$ \\
& 0,5 & $10,2 \mathrm{a}$ & $34 \mathrm{~b}$ \\
& 1,0 & $10,1 \mathrm{a}$ & $108 \mathrm{a}$ \\
& 2,0 & $9,6 \mathrm{a}$ & $85 \mathrm{ab}$ \\
& 4,0 & $7,5 \mathrm{a}$ & $114 \mathrm{a}$ \\
\hline
\end{tabular}

*DMS: diferencia mínima significativa según test de Tukey. Letras distintas indican diferencias significativas entre tratamientos $(\mathrm{P} \leq 0,05)$.

la única variable que presentó interacción significativa en el análisis factorial, pero sólo para el tratamiento de acetato de etilo, siendo este el con menor emergencia en comparación a los demás tratamientos evaluados (Tabla 1). No obstante, la emergencia no se relacionó de modo inversamente proporcional con la mortalidad, ya que a menor porcentaje de mortalidad no necesariamente se registró mayor emergencia de insectos o viceversa. En base a lo mencionado anteriormente se puede inferir que la disminución de individuos en la $\mathrm{F}_{1}$ podría atribuirse a un efecto insectistático de los compuestos del extracto, que inhibirían el desarrollo o modificarían el comportamiento reproductivo de los insectos en lugar de intoxicarlos directamente.

En general la germinación apenas superó el $50 \%$ debido a que se trabajó con maíz destinado a alimentación animalenlugar desemilla certificada. Sin embargo, los menores porcentajes de semillas germinadas (Tabla 2) se obtuvieron en el maíz tratado con metanol al 0,5\% con una germinación de $21,3 \%$, aunque sin diferencia estadística con las otras concentraciones. En los tratamientos con acetato de etilo en concentraciones de $0,5 \mathrm{y}$ $1,0 \%$ se registró un porcentaje de germinación inferior a $50 \%$ aunque tampoco se obtuvieron diferencias significativas entre tratamientos. Con estos antecedentes se puede indicar que la concentración es un factor determinante en la germinación del maíz, principalmente para los tratamientos de hexano y acetato de etilo, tal vez debido a un efecto fitotóxico sobre la semilla. El tratamiento con acetato de etilo al 4,0\% presentó una pérdida de peso (Tabla 2) significativamente menor a los demás tratamientos, lo que podría indicar que ese extracto a dicha concentración podría presentar efecto antialimentario contra $S$. zeamais.

Al comparar los resultados de la presente investigación con otros derivados vegetales evaluados contra S. zeamais (Laynez-Garsaball y Méndez-Natera 2007; Rossi et al. 2012), Sitophilus oryzae L. (Usha y Devanand, 2011) o Zabrotes subsfaciatus Boheman (Tamiru et al. 2016) también reportaron una inhibición en la germinación a medida que aumenta la concentración. Por tanto, el objetivo de encontrar nuevos productos para el control de plagas y enfermedades de los granos almacenados debe incluir el que no afecten la germinación.

\section{Repelencia}

Los resultados del bioensayo indican que el tratamiento en acetato de etilo al 1,0\% presentó la mayor repelencia, registrando según la escala de Bustos et al. (2017) una repelencia muy alta. A su vez el extracto en agua destilada mostró repelencia débil (Tabla 3). Los tratamientos en metanol y hexano no registraron repelencia ya 
Tabla 2. Pérdida de peso y germinación de semillas de maíz tratadas con extractos de P. ostreatus en metanol, hexano y acetato de etilo para el control de adultos de S. zeamais.

Table 2. Weight loss and germination of maize seeds treated with P. ostreatus extracts in methanol, hexane and ethyl acetate for S. zeamais adult control.

\begin{tabular}{cccc}
\hline Tratamiento & $\begin{array}{c}\text { Concentración } \\
(\%)(\mathbf{v} / \mathbf{v})\end{array}$ & $\begin{array}{c}\text { Pérdida de peso* } \\
(\mathbf{\%})\end{array}$ & $\begin{array}{c}\text { Germinación } \\
\mathbf{( \% )}\end{array}$ \\
\hline Metanol & 0,0 & $18,1 \mathrm{a}$ & $25,0 \mathrm{a}$ \\
& 0,5 & $17,8 \mathrm{a}$ & $21,3 \mathrm{a}$ \\
& 1,0 & $16,4 \mathrm{a}$ & $28,7 \mathrm{a}$ \\
& 2,0 & $18,0 \mathrm{a}$ & $27,5 \mathrm{a}$ \\
& 4,0 & $18,3 \mathrm{a}$ & $26,3 \mathrm{a}$ \\
\hline Hexano & 0,0 & $14,4 \mathrm{ab}$ & $52,5 \mathrm{a}$ \\
& 0,5 & $18,1 \mathrm{~b}$ & $43,8 \mathrm{a}$ \\
& 1,0 & $17,8 \mathrm{~b}$ & $41,3 \mathrm{a}$ \\
Ac. etilo & 2,0 & $16,5 \mathrm{ab}$ & $37,5 \mathrm{a}$ \\
& 4,0 & $12,6 \mathrm{a}$ & $36,2 \mathrm{a}$ \\
& 0,0 & $8,8 \mathrm{a}$ & $46,3 \mathrm{a}$ \\
& 0,5 & $13,1 \mathrm{~b}$ & $50,0 \mathrm{a}$ \\
& 1,0 & $11,8 \mathrm{~b}$ & $50,0 \mathrm{a}$ \\
& 2,0 & $11,4 \mathrm{~b}$ & $48,7 \mathrm{a}$ \\
& 4,0 & $8,1 \mathrm{a}$ & $32,5 \mathrm{a}$ \\
\hline
\end{tabular}

*DMS: diferencia mínima significativa según test de Tukey. Letras distintas indican diferencias significativas entre tratamientos $(\mathrm{P} \leq 0,05)$.

Tabla 3. Indice de repelencia (IR) de extractos de P. ostreatus en metanol, hexano y acetato de etilo contra adultos de S. zeamais.

Table 3. Repellency index (RI) of $P$. ostreatus extracts in methanol, hexane and ethyl acetate against S. zeamais adults.

\begin{tabular}{lcc}
\hline Tratamiento & Concentración $(\mathbf{\%}) \mathbf{( v / v )}$ & Índice de Repelencia (IR)* $^{*}$ \\
\hline Metanol & 0,5 & 1,10 \\
& 1,0 & 0,85 \\
& 2,0 & 0,69 \\
4,0 & 1,00 \\
\hline Hexano & 0,5 & 1,31 \\
& 1,0 & 1,23 \\
& 2,0 & 1,07 \\
4,0 & 1,00 \\
\hline Ac. de etilo & 0,5 & 1,18 \\
& 1,0 & 0,0 \\
& 2,0 & 0,64 \\
& 4,0 & 0,61 \\
\hline Agua destilada & 0,5 & 0,99 \\
& 1,0 & 0,91 \\
& 2,0 & 0,77 \\
4,0 & 0,76 \\
\hline
\end{tabular}

$*(\mathrm{IR})=\left(2^{*} \mathrm{G}\right) /\left(\mathrm{G}^{*} \mathrm{P}\right)$ donde $\mathrm{G}=$ porcentaje $(\%)$ de insectos presentes en la placa con tratamiento y $\mathrm{P}=$ porcentaje $(\%)$ de insectos presentes en la placa testigo. $\mathrm{IR}<1$ repelente, $\mathrm{IR}>1$ atrayente, $\mathrm{IR}=1$ neutro 
que los valores del IR son superiores a 1. Estos resultados son similares a estudios realizados con extractos de plantas, como por ejemplo Novo et al. (1997) quienes con extractos de Ch. ambrosioides y L. divaricata en hexano no tuvieron efecto repelente sobre $T$. castaneum, indicando que este solvente no extrae los compuestos con efecto repelente. Sin embargo, Arias et al. (2017) obtuvieron repelencia con aceites esenciales de S. molle contra adultos de S. zeamais registrando valores de IR menores a uno en todas las concentraciones evaluadas, por lo que existe una relación directa en la concentración utilizada y la repelencia; es decir, a mayor concentración mayor es el efecto repelente, como se observa en los tratamientos en acetato de etilo y agua destilada. Por otra parte, Tavares y Vendramim (2005) no constataron efecto repelente en extractos vegetales de Ch. ambrosioides contra adultos de S. zeamais, anteriormente Peterson et al. (1989) sugieren que los compuestos con actividad repelente presentes en este tipo de extracción, no se debe hacer con solventes de alta polaridad, sino que se le debe dar prioridad a los solventes de media o baja polaridad, con el propósito de aislar e identificar las sustancias.

\section{Efecto fumigante}

En la toxicidad por efecto fumigante el único tratamiento efectivo fue el de acetato de etilo con una mortalidad superior a $70 \%$ a partir de

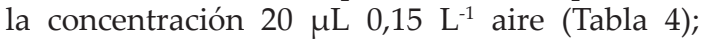
además se observó una tendencia de incremento de la mortalidad en la medida que aumenta la concentración del extracto. Considerando el criterio de Lagunes (1994), se puede indicar que de los cuatro extractos evaluados, sólo acetato de etilo se puede clasificar como prometedor. El extracto en acetato de etilo presenta diferencias significativas con metanol, hexano y agua destilada, los que registraron valores de mortalidad inferiores a 5\%. Estos resultados son similares a los obtenidos con otros compuestos vegetales, como los aceites esenciales. Por ejemplo Pizarro et al. (2013) registraron toxicidad por fumigación mayor a $90 \%$ con los tratamientos de

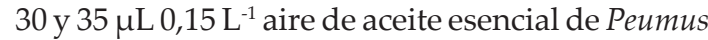
boldus Molina, y Bustos et al. (2017) registraron un $80 \%$ de mortalidad en concentraciones de

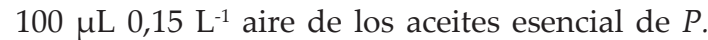
boldus, Laurelia sempervirens (Ruiz \& Pav.) Tul. y Laureliopsis philippiana (Looser) Schodde. Sin embargo, se debe considerar que el extracto sería mejor opción que el aceite esencial debido a que se obtiene mayor rendimiento a partir del material vegetal, y que para obtener aceite esencial se necesita mayor cantidad de follaje y tiempo de extracción. Finalmente, en base al bioensayo realizado en la presente investigación se puede señalar que el extracto de $P$. ostreatus en acetato de etilo es prometedor como insecticida fumigante contra $S$. zeamais, pero debe ser validado con bioensayos en bodega.

$\mathrm{Al}$ calcular la Concentración Letal 50\% $\left(\mathrm{CL}_{50}\right)$ y $90 \%\left(\mathrm{CL}_{90}\right)$ para el extracto en acetato de etilo se obtuvo una $\mathrm{CL}_{50}$ de $18,3 \mu \mathrm{L} 0,15 \mathrm{~L}^{-1}$ aire (equivalente a $122 \mu \mathrm{L} \mathrm{L}^{-1}$ aire) y de $22,1 \mu \mathrm{L} 0,15$ $\mathrm{L}^{-1}$ aire (equivalente a $147 \mu \mathrm{L} \mathrm{L}^{-1}$ aire) cuando se requiere controlar el $90 \%$ de los insectos. Aunque no existen estudios de extractos de hongos comestibles para el control $S$. zeamais, en el aceite esencial de $P$. boldus se ha reportado una $\mathrm{CL}_{50}$ de $115,5 \mu \mathrm{L}$ aceite esencial $\mathrm{L}^{-1}$ aire (Bustos et al., 2017), resultado muy similar al obtenido

Tabla 4. Efecto fumigante de extractos de $P$. ostreatus en metanol, hexano y acetato de etilo contra adultos de $S$. zeamais.

Table 4. Fumigant effect of $P$. ostreatus extracts in methanol, hexane and ethyl acetate against $S$. zeamais adults.

\begin{tabular}{lcccc}
\hline \multicolumn{5}{c}{ Mortalidad (\%)* } \\
\hline $\begin{array}{l}\text { Concentración } \\
\mu \mathrm{L} 150 \mathrm{~mL}^{-1} \text { aire }\end{array}$ & Metanol & Hexano & Acetato de etilo & Agua destilada \\
\hline Testigo & $0,0 \mathrm{a}$ & $0,0 \mathrm{~b}$ & $0,0 \mathrm{~b}$ & $0,0 \mathrm{a}$ \\
5 & $10,0 \mathrm{aB}$ & $22,5 \mathrm{aA}$ & $5,0 \mathrm{bAB}$ & $5,0 \mathrm{aAB}$ \\
10 & $5,0 \mathrm{aB}$ & $12,5 \mathrm{abA}$ & $2,5 \mathrm{bA}$ & $0,0 \mathrm{aA}$ \\
15 & $0,0 \mathrm{aA}$ & $2,5 \mathrm{abA}$ & $7,5 \mathrm{bA}$ & $0,0 \mathrm{aA}$ \\
20 & $2,5 \mathrm{aB}$ & $0,0 \mathrm{bB}$ & $75,0 \mathrm{aA}$ & $2,5 \mathrm{aB}$ \\
25 & $2,5 \mathrm{aB}$ & $10,0 \mathrm{abB}$ & $97,5 \mathrm{aA}$ & $2,5 \mathrm{aB}$ \\
30 & $0,0 \mathrm{aB}$ & $0,0 \mathrm{bB}$ & $100,0 \mathrm{aA}$ & $5,0 \mathrm{aB}$ \\
\hline
\end{tabular}

"DMS: diferencia mínima significativa según test de Tukey. Letras minúsculas distintas indican diferencias significativas entre concentraciones $(\mathrm{P} \leq 0,05)$. Letras mayúsculas distintas indican diferencias significativas entre tratamiento $(\mathrm{P} \leq 0,05)$. 
con el extracto en acetato de etilo. Aunque estos resultados son mayores a los de Salgado et al. (2012) quienes obtuvieron valores de $\mathrm{CL}_{50}$ para el control de $S$. zeamais de 0,07 y $0,63 \mu \mathrm{L} \mathrm{cm}^{-2}$ con el aceite esencial de Piper crassinervum Kunth.

\section{Antixenosis (No preferencia)}

Los resultados de antixenosis (no preferencia) indican que al comparar los diferentes extractos con el testigo casi todos los tratamientos evaluados presentan una menor preferencia de S. zeamais (Tabla 5). La única excepción fueron las concentraciones de 0,25 y $0,5 \%$ del extracto en agua destilada que registraron valores de IR $>$ 1indicando atracción. Según Lizarazo et al. (2008) el agua sólo extrae compuestos de alta polaridad, como glucósidos, la mayoría de los cuales presentan una baja actividad biológica. Además, es común que en extractos con baja actividad biológica las menores concentraciones evaluadas muestran resultados no consistentes. En los tratamientos con metanol, hexano y acetato de etilo se observa una disminución del IR a medida que las concentraciones aumentan, no registrándose índices $>1$. Al respecto, varios estudios han demostrado actividad antixenótica de compuestos vegetales no tan sólo contra $S$. zeamais, sino también sobre otras especies de insectos como Plutella xylostella L., Aedes aegypti L (Diptera; Culicidae) (Choochote et al., 2007) y Trialeurodes vaporariorum Westwood (Hemiptera; Aleyrodidae) (Dehghani y Ahmadi, 2013). A pesar que no existen estudios de extractos de hongos comestibles para el control de $S$. zeamais, la antixenosis se caracteriza por la menor utilización del huésped por el insecto, ya sea para la alimentación, oviposición y/o refugio (Fancelli et al., 2005). En estudios anteriores Obeng-Oferi y Reichmuth (1997), señalan que en el caso de las plagas de productos almacenados la acción de disminuir la preferencia de alimentos de insectos por el maíz tratado puede ser una herramienta potencial para prevenir la infestación de los granos almacenados.

Finalmente, se puede señalar que los resultados obtenidos en los bioensayos de toxicidad por fumigación, repelencia y antixenosis permiten considerar al extracto de $P$. ostreatus como una alternativa prometedora para el control de adultos de $S$. zeamais pero que debe ser validada con posterioridad en bioensayos de bodega.

\section{CONCLUSIONES}

Con base en los resultados obtenidos en la presente investigación se puede concluir que el extracto de $P$. ostreatus en acetato de etilo presenta actividad biológica prometedora como repelente, fumigante y antixenótico para el control de $S$. zeamais.

Tabla 5. Efecto antixenótico (No preferencia) de extractos de P. ostreatus con solventes de distinta polaridad (hexano $>$ metanol $>$ acetato de etilo $>$ agua destilada.) contra adultos de S. zeamais.

Table 5. Antixenotic effect (No preference) of $P$. ostreatus extracts with different polarity extracts (hexane $>$ methanol $>$ ethyl acetate $>$ distilled water) against $S$. zeamais adults.

\begin{tabular}{ccccc}
\cline { 2 - 5 } & \multicolumn{4}{c}{ Índice de Repelencia (IR)* } \\
\hline $\begin{array}{c}\text { Concentración } \\
\text { (\%) (v/v) }\end{array}$ & Metanol & Hexano & Ac. etilo & Agua destilada \\
\hline 0,125 & 0,93 & 0,88 & 0,93 & 0,90 \\
0,25 & 0,89 & 0,86 & 0,90 & 1,10 \\
0,5 & 0,82 & 0,82 & 0,88 & 1,08 \\
1,0 & 0,78 & 0,79 & 0,83 & 0,68 \\
2,0 & 0,71 & 0,53 & 0,76 & 0,85 \\
4,0 & 0,56 & 0,47 & 0,69 & 0,56 \\
8,0 & 0,44 & 0,43 & 0,64 & 0,90 \\
\hline
\end{tabular}

$*(I R)=(2 * G) /\left(G^{*} P\right)$ donde $G=$ porcentaje $(\%)$ de insectos presentes en la placa con tratamiento y $\mathrm{P}=$ porcentaje $(\%)$ de insectos presentes en la placa testigo. $\mathrm{IR}<1$ no preferencia, $\mathrm{IR}>1$ preferencia, IR $=1$ neutro. 


\section{LITERATURA CITADA}

Abbott, W.S. 1925. A method of computing the effectiveness of an insecticide. J. Econ. Entomol. 18(2):265-267. doi: 10.1093/ jee/18.2.265a

Almeida, F.A., P.J. da Silva, V. Queiroga, A. Neto, N. Olivier, y A.B. Rojas. 2014. Eficiencia de extractos vegetales como insecticidas sobre Sitophilus zeamais en granos almacenados. Rev. Cienc. Téc. Agropec. 23(2):57-62. doi: 10.1590/S1413-70542006000400029

Arias, J., G. Silva, I. Figueroa, S. Fisher, A. RoblesBermúdez, J.C. Rodríguez-Maciel, y A. Lagunes-Tejada. 2017. Actividad insecticida, repelente y antialimentaria del polvo y aceite esencial de frutos de Schinus molle L. para el control de Sitophilus zeamais (Motschulsky). Chilean J. Agric. Anim. Sci. 33(2):93-104. doi: 10.4067/S0719-38902017005000301

Bustos, G., G. Silva, S. Fisher, I. Figueroa, A. Urbina, y J.C. Rodríguez. 2017. Repelencia de mezclas de aceites esenciales de boldo, laurel chileno, y tepa contra el gorgojo del maíz. Southwest. Entomol. 42(2):551-563. doi: 10.3958/059.042.0224.

Caldas, A.P. 2012. Optimización, escalamiento y diseño de una planta piloto de extracción sólido líquido. Tesis, Ingeniera Química. Universidad de Cuenca, Facultad de Ciencias Químicas, Escuela de Ingeniería Química. Cuenca, Ecuador.

Carrillo, J.D., R.O. Méndez, R. Gutiérrez, C.A. Reyes, y P.P. Miranda. 2017. Actividad antibacteriana de Pleurotus ostreatus [en línea]. ResearchGate, Alemania. <https://www. researchgate.net/publication/322235757 ACTIVIDAD_ANTIBACTERIANA_ DE_Pleurotus_ostreatus>. [Consulta: $1 \overline{6}$ septiembre 2018].

Choochote, W., U. Chaithong, K. Kamsuk, A. Jitpakdi, P. Tippawangkosol, B. Tuetun, et al. 2007. Repellent activity of selected esencial oils against Aedes aegypti. Fitoterapia 78(5): 359-364. doi: 10.1016/j.fitote.2007.02.006

Chu, S.S., J.F. Hu, and Z.L. Liu. 2011. Composition of essential oil of Chinese Chenopodium ambrosioides and insecticidal activity against maize weevil, Sitophilus zeamais. Pest Manag. Sci. 67(6):714-718. doi: 10.1002/ps.2112. Epub $2011 \mathrm{Feb} 24$.

Cruzat, M., G. Silva, H. Serri, y R. Hepp. 2009. Protección de ocho cultivares de trigo con polvo de Peumus boldus Molina contra Sitophilus zeamais Motschulsky. Idesia 27(2):39-46. doi: 0.4067/S071834292009000200005
Dehghani, M., and K. Ahmadi. 2013. Antioviposition and repellence activities of essential oils and aqueous extracts from five aromatic plant against greenhouse whitefly Trialeurodes vaporarorium Westwood (Homoptera: Aleyrodidae). Bulg. J. Agric. Sci. 19(4):691-696. doi: 10.5530/pc.2013.2.2

Devi, S.R., A. Thomas, K.B. Rebijith, and V.V. Ramamurthy. 2017. Biology morphology and molecular characterization of Sitophilus oryzae and S. zeamais (Coleoptera: Curculionidae). J. Stored Prod. Res. 73:135-141. doi: 10.1016/j. jspr.2017.08.004

Fancelli, M., J.D. Vendramin, R.T.S. Frighetto, e A.L. Lourenção. 2005. Exsudato glandular de genotipo de tomateiro e desenvolvimiento de Bemisa tabaci (Genn) (Sternorryncha: Aleyrodidae) biótipo B. Neotrop. Entomol. 34(4):659-665. doi: 10.1590/S1519566X2005000400017

Ganash, M.A., T.M. Abdel, and A.M. Reyad. 2016. Pleurotus ostreatus as a biodegradator for organophosphorus insecticide matathion. J. Environ. Anal. Toxicol. 6(369). doi:10.4172/2161-0525.100036. Disponible en https://pdfs.semanticscholar.org $/ 5 \mathrm{fcb} /$ c27fda209d896511ef141f6bd5ec02fcbd88.pdf. (Consulta 15 Octubre 2019).

García-Lara, S., C. Espinosa, y D.J. Bergvinson. 2007. Manual de plagas en granos almacenados y tecnologías alternas para su manejo y control. Centro Internacional de Mejoramiento de Maíz y Trigo, México D.F., México.

Halstead, D.G.H. 1963. External sex differences in stored-products Coleoptera. Bull. Entomol. Res. 54(1):119-134. doi:10.1017/ S0007485300048665

Hernández-Lambraño, R., N. Pájaro-Castro, K. Caballero-Gallardo, E. Stashenko, and J. Olivero-Verbel. 2015. Essential oils from plants of the genus Cymbopogon as natural insecticides to control stored product pests. J. Stored Prod. Res. 62:81-83. doi: 10.1016/j. jspr.2015.04.004

Hou, C.-L., J.-B. Wang, H. Wu, J.-Y. Liu, Z.Q. Ma, J.-T Feng, et al. 2016. Molecular cloning and expression analysis of cytochrome $c$ oxidase subunit II from Sitophilus zeamais. Biochem. Biophys. Res. Commun. 478(4):1660-1666. doi: 10.1016/j.bbrc.2016.08.176

Kim, S., K. Jaesson, and I. Park. 2013. Fumigant toxicity of Apiaceae essential oils and their constituents against Sitophilus oryzae and their acetylcholinesterase inhibitory activity. Asia-Pac. Entomol. 16:443-448. doi: 10.1016/j. aspen.2013.07.002 
Lagunes, A. 1994. Extractos y polvos vegetales y polvos minerales para el combate de plagas del maíz y del frijol en la agricultura de subsistencia. Memoria. Colegio de Postgraduados en Ciencias Agrícolas. Texcoco, México.

Laynez-Garsaball, J.A., y J.R. Méndez-Natera. 2007. Efectos de extractos acuosos de la maleza Cyperus rotundus L. (Cyperaceae) sobre la germinación de semillas y crecimiento de plántulas de maíz (Zea mays L.) cv. Pioneer 3031. Rev. Peru. Biol. 14(1):5560. doi: 10.15381/rpb.v14i1.1759

Lizarazo, K., C. Mendoza, y R. Carrero. 2008. Efecto de extractos vegetales de Polygonum hydropiperoides, Solanum nigrum y Calliandra pittieri sobre el gusano cogollero (Spodoptera frugiperda). Agron. Colomb. 26(3):427-434.

Mazzonetto, F., e J.D. Vendramim. 2003. Efeito de pós de origem vegetal sobre Acanthoscelides obtectus (Say) (Coleoptera: Bruchidae) em feijão armazenado. Neotrop. Entomol. 32(1):145-149. doi: 10.1590/S1519566X2003000100022

Moreno, E. 1996. Análisis físico y biológico de semillas agrícolas. Universidad Nacional Autónoma de México, México D.F., México.

Novo, R.J., A. Viglianco, y M. Nassetta. 1997. Actividad repelente de diferente extracto vegetales sobre Tribolium castaneum (Herbst). AgriScientia 16:31-36. doi: 0.31047/1668.298x. v14.n0.2524

Obeng-Ofori, D., and C. Reichmuth. 1997. Bioactivity of eugenol, a major component of essential oil Ocimum suave (Wild.) against four species of stored-product Coleoptera. Int. J. Pest Manag. 43(1):89-94. doi: 10.1080/096708797229040.

Ortiz, A., G. Silva, A. Urbina, N. Zapata, J.C. Rodríguez, y A. Lagunes. 2012. Bioactividad del polvo de tepa (Laureliopsis philippiana (Looser) Shodde) para el control de Sitophilus zeamais Motschulsky control in laboratory. Chilean J. Agric. Res. 72(1):68-73. doi: 10.4067/S0718-58392012000100011

Pérez, F., G. Silva, M. Tapia, y R. Hepp. 2007. Variación anual de las propiedades insecticidas de Peumus boldus sobre Sitophilus zeamais. Pesq. Agropec. Bras. 42(5):633-639. doi: 10.1590/S0100-204X2007000500004

Peterson, G.S., M.A. Kandil, M.D. Abdallah, and A.-A.A. Farag. 1989. Isolation and characterisation of biologically-active compounds from some plant extracts. Pestic. Sci. 25(4):337-342.
Pizarro, D., G. Silva, M. Tapia, J.C. Rodríguez, A. Urbina, A. Lagunes, et al. 2013. Actividad insecticida del polvo de Peumus boldus Molina (Monimiaceae) contra Sitophilus zeamais Motschulsky (Coleoptera: Curculionidae). Bol. Latinoam. Caribe Plant. Med. Aromát. 12(4):420-430. doi: 10.1002/ps.2780250403.

Pohleven, J., J. Brzin, L. Vrabec, A. Leonardi, A. Čokl, B. Štrukelj, et al. 2011. Basidiomycete Clitocybe nebularis is rich in lectins with insecticidal activities. Appl. Microbiol. Biotechnol. 91(4):1141-1148. doi: 10.1007/ s00253-011-3236-0

Rahman, M.F., M.R. Karim, M.J. Alam, M.F. Islam, M.R. Habib, M.B. Uddin et al. 2011. Insecticidal effect of oyster mushroom (Pleurotus ostreatus) against Tribolium castaneum (Herbst). NPAIJ 7(4):187-190.

Regnault-Roger, C. 1997. The potencial of botanical essential oils for insect pest control. Int. Pest Manag. Rev. 2(1):25-34. doi: 10.1023/A:1018472227889.

Rossi, E., S. Cosimi and A, Loni. 2012. Bioactivity of essential oils from mediterranean plants: insecticidal properties on Sitophilus zeamais and effects on seed germination. J. Entomol. 9:403-412. doi: http://dx.doi.org/10.3923/ je.2012.403.412

Salgado, N., L.R. D'Antonino, y A. Soto. 2012. Aceite esencial de Piper cressinervum para el control de Sitophilus zeamais (Coleoptera: Curculionidae). Bol. Cient. Mus. Hist. Nat. 16(1):99-107.

Silva, G., A. Lagunes, y J. Rodríguez. 2003. Control de Sitophilus zeamais (Coleoptera: Curculionidae) con polvos vegetales solos y en mezcla con carbonato de calcio en maíz almacenado. Cienc. Investig. Agrar. 30(3):153160. doi: 10.1590/S0100-204X2005001000002

Tamiru, A. T. Bayih and M. Chimdessa. 2016. Synergistic bioefficacy of botanical insecticides against Zabrotes subfasciatus (Coleoptera: Bruchidae) a major storage pest of common ben. J. Fertil. Pestic. 7(2):1-8. doi: 10.4172/2471-2728.1000171

Tavares, M.A.G.C. e J.D. Vendramim. 2005. Bioatividade da Erva-de-Santa-Maria, Chenopodium ambrosioides L., sobre Sitophilus zeamais Mots. (Coleoptera: Curculionidae). Neotrop. Entomol. 34(2):319-323. doi: 10.1590/S1519-566X2005000200021

Usha, P.R. and P. Devanad. 2011. Efficiency of different plant foliar extracts on grain protection and seed germination in maize. Res. J. Seed Sci. 4(1):1-14. doi: 10.3923/ rjss.2011.1.14. 
Usseglio, V.L., R.P. Pizzolito, C. Rodriguez, M.P. Zunino, J.A. Zygadlo, V.A. Areco, et al. 2017. Volatile organic compound from interaction betwen Fusarium verticillioides and maize kernels as a natural repellents of Sitophilus zeamais. J. Stored Prod. Res. 73:109-114. doi: 10.1016/j.jspr.2017.08.001

Wale, M., and H. Assegie. 2015. Efficacy of castor vean oil (Ricinus communis L.) against maize weevils (Sitophilus zeamais Mots.) in northwestern Ethiopia. J. Stored Prod. Res. 63:38-41. doi: 10.1016/j.jspr.2015.05.006

Zettler, J.L., and F.H. Arthur. 2000. Chemical control of stored product insect with fumigants and residual treatments. Crop Prot. 19(8-10):577-582. doi: 10.1016/S02612194(00)00075-2 